BMJ Open

Diabetes

Research

\& Care

\section{Patients' perspectives on social and goal-based comparisons regarding their diabetes health status}

\author{
William Martinez, ${ }^{1}$ Kenneth A Wallston, ${ }^{2}$ David G Schlundt, ${ }^{3}$ Gerald B Hickson, ${ }^{4}$ \\ Kemberlee R Bonnet, ${ }^{3}$ Ricardo J Trochez, ${ }^{1}$ Tom A Elasy ${ }^{1}$
}

To cite: Martinez W, Wallston KA, Schlundt DG, et al. Patients' perspectives on social and goal-based comparisons regarding their diabetes health status. BMJ Open Diab Res Care 2018;6:e000488. doi:10.1136/ bmjdrc-2017-000488

- Additional material is published online only. To view please visit the journal online (http://dx.doi.org/10.1136/ bmjdrc-2017-000488).

Received 19 0ctober 2017 Revised 18 April 2018 Accepted 25 April 2018

\section{Check for updates}

${ }^{1}$ Division of General Internal Medicine and Public Health, Vanderbilt University Medical Center, Nashville, Tennessee, USA

${ }^{2}$ Vanderbilt University School of Nursing, Nashville, Tennessee, USA

${ }^{3}$ Department of Psychology, Vanderbilt University, Nashville, Tennessee, USA

${ }^{4}$ Quality, Safety and Risk

Prevention, Vanderbilt University Medical Center, Nashville, Tennessee, USA

Correspondence to Dr William Martinez; william.martinez@vanderbilt. edu

\section{ABSTRACT}

Objective Social comparisons (ie, self-evaluation in comparison with others) influence patients' perspectives of their disease and may impact motivation and health behavior; however, little is known about patients' perspectives toward receiving such information in a clinical context (eg, from their doctor's office or health system). This study aims to understand patients' perspectives and anticipated responses to receiving social comparison information regarding measures of their diabetes-related health status (eg, $\mathrm{A} 1 \mathrm{C})$ and how receiving such information would compare with goal-based comparisons (ie, selfevaluation in comparison with goal).

\section{Research design and methods We conducted} semistructured interviews with 25 patients with type 2 diabetes mellitus (T2DM) regarding social and goal-based comparisons involving their diabetes health status and qualitatively analyzed interviews for themes.

Results We identified seven major themes: self-relevance, motivation, self-concept, emotions, information seeking, medical care, and self-care. Participants commonly anticipated increased motivation and improved health behaviors in response to both social and goal-based comparisons. Subthemes unique to social comparisons included belief that this information would be motivating by engaging some patients' competitiveness, perception that this information was more 'personalized' than comparisons with a standard goal (eg, $\mathrm{A} 1 \mathrm{C}<7)$, and desire to learn from individuals similar to oneself who were doing better. Conclusions Our findings provide significant insights into the anticipated response of patients with T2DM to receiving social and goal-based comparison information regarding their diabetes health status. Providing patients with diabetes with social and goal-based comparison information may affect motivation, mood, and self-concept in ways that may improve or sustain diabetes self-care behaviors for some patients.

\section{INTRODUCTION}

In the USA, diabetes is the leading cause of kidney failure, non-traumatic lower limb amputations, and new cases of blindness, and a contributor to heart disease and stroke. ${ }^{1}$ Patients with diabetes can significantly reduce the risk of these complications through medication adherence, disease monitoring, preventive health services, exercise,

\section{Significance of this study}

What is already known about this subject?

- A growing literature demonstrates the important role of social comparisons (ie, self-evaluation in comparison with others) on patients' perspectives of their disease and shaping motivation and behavior, but patients' perspectives toward receiving such information in a clinical context (eg, from their doctor's office or health system) are unknown.

\section{What are the new findings?}

- We found that social comparison information may be viewed as more personalized, uniquely engage some patients' competitiveness, and promote information seeking when compared with goal-based comparisons (ie, self-evaluation in comparison with goal status) and that providing patients with diabetes with social comparison information regarding measures of their diabetes health status (eg, A1C) may affect motivation, mood, and self-concept in ways that may improve or sustain diabetes self-care behaviors for some patients.

How might these results change the focus of research or clinical practice?

- Our findings suggest that further research is needed to determine how to best share social comparison information with patients, which patients are most likely to benefit, and whether patients' anticipated responses match actual responses to this information as well as the impact of sharing this information on cognitive, behavioral, and clinical outcomes.

diet, and maintaining a healthy weight. ${ }^{2}$ However, these self-care behaviors can feel burdensome to many patients and are challenging to implement and sustain. ${ }^{3}$

While many interventions have focused on enhancing and maintaining patients' motivation for diabetes self-care behaviors, many limitations and gaps exist. ${ }^{45}$ In particular, while some interventions have focused on increasing patients' social supports via 
peer coaching and other peer-led programs, ${ }^{6-8}$ there has been less attention on other social influences on self-care behaviors. ${ }^{9}$ Some interventions have focused on motivating patients by increasing their access to and knowledge of their own diabetes-related health data (eg, hemoglobin $\mathrm{A} 1 \mathrm{C}$ and blood pressure) relative to established goals (eg, hemoglobin $\mathrm{A} 1 \mathrm{C}<7)$ that are associated with improved diabetes outcomes. These goal-based comparisons alone have limited effects on increasing the confidence and motivation necessary to improve patients' diabetes self-management. ${ }^{10-12}$

A growing literature demonstrates the important role of social comparisons (ie, self-evaluation in comparison with others) on patients' perspectives of their disease and shaping motivation and behavior. ${ }^{13-16}$ Several studies, including one of patients with diabetes, demonstrate that patients commonly and spontaneously compare themselves to other patients, and one study demonstrated that patients with diabetes can experience increases in motivation for diabetes self-management when they compare themselves to a patient who is doing better than they are (ie, upward comparison) as well as when they compare themselves to a patient who is doing worse (ie, downward comparison). ${ }^{15}$ Upward comparisons can inspire individuals to take action toward a positive outcome, while downward comparisons can provide positive reinforcement and inspire individuals to take action to avoid a negative outcome. ${ }^{1516}$ The combination of both social and goal-based comparison information may be more motivating than either alone. ${ }^{17} 18$

Despite the potential of social comparisons to positively influence diabetes self-care behaviors and compliment goalbased comparisons, little is known about patients' perspectives toward receiving such information in a clinic context (ie, from their doctor's office or healthcare system). This study aims to understand patients' perspectives and anticipated responses to receiving social comparison information regarding measures of their diabetes-related health status (eg, A1C, cholesterol and eye examination in the last year) and how receiving such information would compare to goal-based comparisons.

\section{METHODS}

Study design

From November 2015 to February 2016, we conducted individual qualitative semistructured interviews of patients with type 2 diabetes mellitus (T2DM). Qualitative interviews are the preferred methodology to gain in-depth understanding of individual perspectives or responses to specific topics or stimuli. ${ }^{19}$ Study participants were recruited from the Vanderbilt Adult Primary Care (VAPC) clinic at Vanderbilt University Medical Center (VUMC) in Nashville, Tennessee.

\section{Setting}

The VAPC clinic cares for about 25000 unique patients annually of which about $4500(18 \%)$ have diabetes. The clinic is composed of 36 part-time and full-time attending physicians and 31 nurses and medical assistants. All clinical data are entered into an electronic medical record, and patients are provided access to a portion of their clinical data through a patient web portal. The clinic's patient population is $60 \%$ Caucasian (non-Hispanic), $30 \%$ African-American, $8 \%$ Hispanic, and 2\% Asian and closely matches the racial demographics of the Nashville population. ${ }^{20}$ The majority of clinic patients $(74 \%)$ are privately insured, $24 \%$ are on Medicare/Medicaid, and less than $2 \%$ are uninsured.

\section{Sampling and recruitment}

Eligible patients were identified automatically using VUMC's Subject Locator to query the electronic health records (including diagnosis codes) associated with patients with upcoming clinic appointments for discrete inclusion and exclusion criteria. English-speaking patients aged 21 years and older with T2DM (identified by International Statistical Classification of Diseases, Ninth/Tenth Revision codes) and prescribed at least one antihyperglycemic medication were eligible to participate. Since a patient's health system or doctor's office is uniquely well positioned to provide patients with social comparison information (eg, patient's A1C in comparison with the average A1C of other patients who are characteristically similar), we focused our study on receiving such information in that setting and limited recruitment to patients with an established relationship with a primary care physician within the clinic (at least two visits within the preceding 24 months). Patients with cognitive impairments and nursing home residents were excluded. All eligible patients with upcoming clinic appointments were mailed a letter describing the study and asked to contact a research coordinator if they were interested in participating. Letters were sent to 841 patients with upcoming appointments on a rolling basis over 4 months. Interested patients $(n=68)$ contacted the research coordinator who explained the study, answered questions, and confirmed eligibility. Thirty-five patients wished to participate and were offered the opportunity to schedule a study interview on predetermined dates based on interviewer availability. Whenever possible, patients were offered an interview on the day of their upcoming clinic appointment. Patients who were unavailable on the predetermined interview dates were contacted on a rolling basis as additional interview dates became available until thematic saturation was reached.

We aimed to enroll a mix of patients who reflected a broad range of experiences with diabetes including patients who use and do not use insulin, with currently well controlled and poorly controlled diabetes, and with and without diabetes-related comorbidities. This occurred naturally as study recruitment progressed, thus purposive sampling was not required.

All participants provided written informed consent. Patients received $\$ 30$ as compensation for their participation. 


\section{Data collection}

Enrolled patients were asked to complete a short, self-administered questionnaire prior to their interview. The questionnaire included basic demographic questions along with the following measures: three-item health literacy screen ${ }^{21}$; five-item diabetes numeracy scale ${ }^{22}$; and the Perceived Diabetes Self-Management Scale. ${ }^{23}$ In addition, clinical data including the most recent hemoglobin A1C, medication list, and problem list were extracted from participant's medical record.

Each participant took part in a one-on-one, in-person, semistructured interview with a study interviewer. Two individuals (WM and TLI) conducted the interviews under the supervision of an expert in qualitative methods (DGS). Interviewers used an interview guide (online supplementary appendix 1) consisting of openended questions designed to elicit participants' perspectives and anticipated responses to receiving social and goal-based comparison information regarding their diabetes health status. Interviews took place in a private setting (ie, a small conference room in close proximity to the clinic) and averaged $56 \mathrm{~min}$ in duration. Interviews were audio-recorded to allow accurate transcription. Preliminary analysis of interviews began after the first five interviews were completed, and consistent with established qualitative research methods, additional patients were recruited and interviewed until thematic saturation was achieved. ${ }^{24}$ Criterion for determining saturation was no new themes emerging in the preceding five interviews. $^{25}$

\section{Data analysis}

Audio files of interviews were transcribed by a professional transcription service (http://www.rev.com/transcription). Transcripts were checked for accuracy and identifying information was removed. A coding scheme was developed by two of the authors (DGS and KRB), who did not participate in the interviews, using a combined approach that used a priori and inductive processes and consistent with methods described in Miles et al's study. ${ }^{26}$ A 'start list' of codes grouped into broad categories was developed based on the interview guide and research aims. The first five transcripts were then open coded, and initial themes 'grounded' in the transcripts were identified and used to refine the start list into a preliminary codebook that provided clear operational definitions for each code. ${ }^{27}$ This initial set of codes was tested on each subsequent interview transcript; codes were further revised in an iterative manner using the constant comparison method between and within transcripts, and additional 'grounded' axial codes were applied. ${ }^{27}$ The final coding scheme was established, and two coders coded all interview transcripts. The coders applied codes to participants' statements (primary unit of analysis) and were permitted to apply multiple codes to a single statement as applicable. Once the coding was completed, the codes were organized into the main themes. Representative excerpts were selected from interview transcripts and edited slightly for grammar and spelling. We used NVivo 10 (QSR International, Burlington, Vermont) to assist with qualitative analysis.

Based on a review of the literature, ${ }^{13-16 ~ 28-33}$ we developed an a priori conceptual model consisting of four domains: (1) type of comparison; (2) cognitive effects; (3) behavioral effects; and (4) effect modifiers to organize our analysis. Qualitative analysis of interview transcripts identified relevant themes and subthemes within each of the four general domains, which were subsequently added to the final model (figure 1).

Descriptive statistics were used to characterize the study participants. Statistical analyses were performed using SAS V.9.4.

\section{RESULTS}

\section{Participants}

We interviewed a total of 25 patients, at which time we had reached saturation. Table 1 shows the characteristics of study participants. Participants had a mean age of 65 years (range 45-81), 52\% were women, $68 \%$ were white, the mean A1C was 7.5 (range 5.6-12.6), 44\% had an A1C above 7 (range 7.1-12.6), and 52\% were currently being treated with insulin. Participants with a college degree or higher made up 52\% of our sample. Many participants had comorbid conditions: $71 \%$ had hyperlipidemia, $60 \%$ had hypertension, and $20 \%$ had atherosclerotic cardiovascular disease.

\section{Themes}

We identified seven major themes: self-relevance, motivation, self-concept, emotions, information seeking, medical care, and self-care. Motivation, self-concept, and emotions represent potential cognitive effects of comparison information; information seeking, medical care and self-care represent potential downstream behavioral effects; and self-relevance represents a potential effect modifier (see conceptual model, figure 1). Table 2 lists the major themes and their associated subthemes including those that were unique to participants' statements regarding social comparisons. Each of the major themes is presented below along with illustrative quotes.

\section{Self-relevance}

Most participants $(n=14)$ described goal-based comparisons of their diabetes health status as important to their understanding of their diabetes management (subtheme).

It's my fault [my blood sugar is higher than goal]. [I] should have been back on a diet. I should have stayed out of that pie.

Only two participants expressed disinterest and described relying primarily on a subjective sense of well-being.

I know I can look up recommendations or whatever, but if I'm doing good I don't need to know that. I mean, I'm satisfied. 


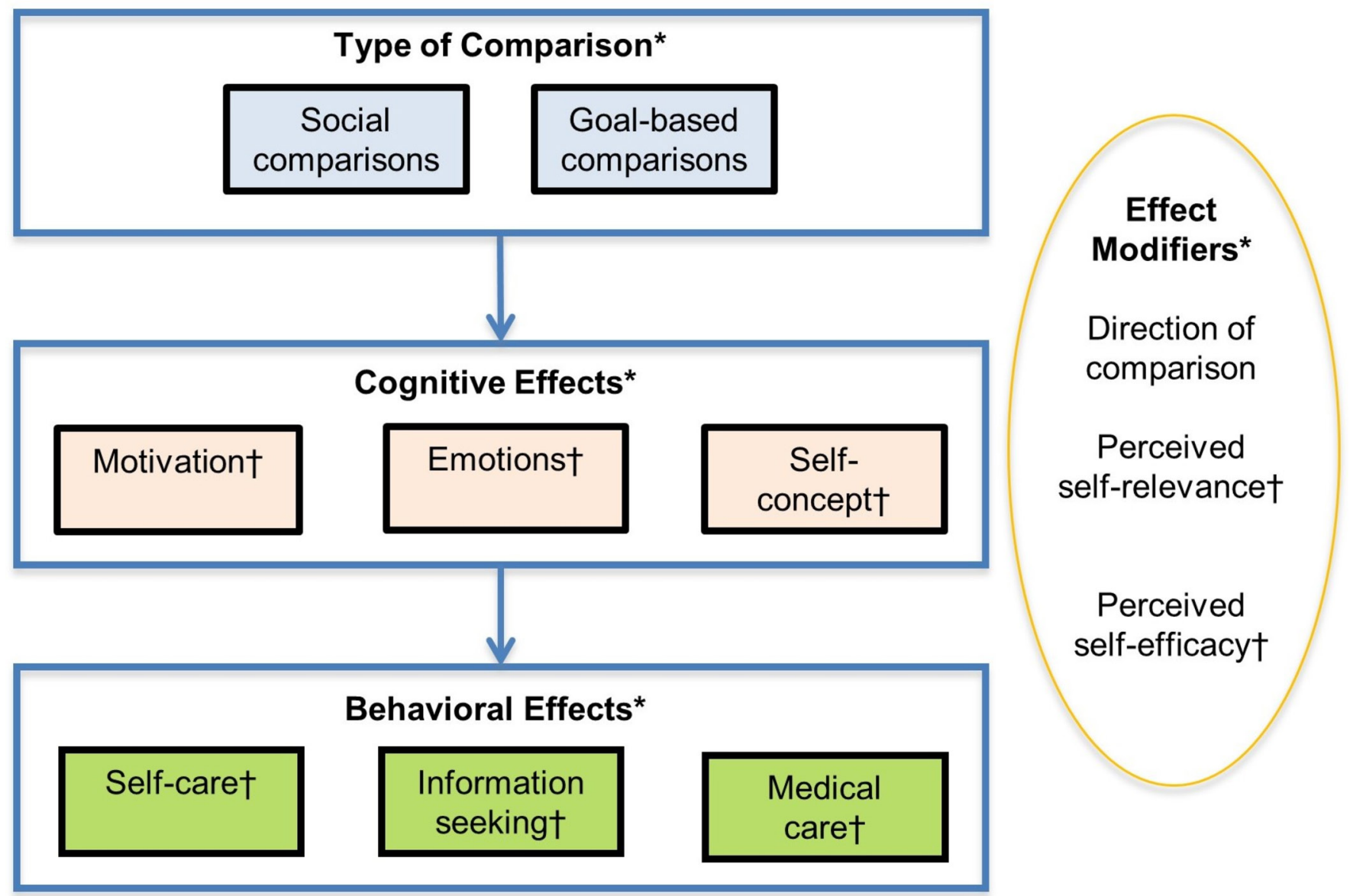

Figure 1 Final conceptual model of social and goal-based comparisons of diabetes health status. *A priori domains used to organize analysis. †Relevant themes and subthemes identified from qualitative analysis of interview transcripts and subsequently added to the model.

Even more participants $(n=17)$ expressed a desire for social comparison information regarding their diabetes health status.

I can see how that could be very helpful [...] it would be interesting to see how other diabetics fall, [...] it might also make me a little more appreciative if I'm doing a lot better.

Only four patients described disinterest in social comparison information and often described their lack of interest in terms of the uniqueness of each person's experience (subtheme).

I'm not curious about how other people are because I think any disease or any illness affects everybody differently in one form or another.

Thirteen participants anticipated that being compared with others who shared certain characteristics would make the information more relevant to them. Participants specifically commented on the importance of being compared with others who shared similar characteristics including one or more of the following: same age group, gender, body type, medications, and racial and ethnic group.
I would think that it would be more important that it be women because everybody is different and men's bodies are totally different from women's bodies and they're going to react in a certain way that a woman would not, so I would rather compare myself to a group of women that are in the same category, say the same age.

Some participants who described a desired for social comparison information commented on the more personalized nature of the information (subtheme) and opportunity to assess their condition relative to similarly matched others (subtheme).

I would be happy to have that information. To see how my situation compares to other people. Maybe if there was some things in there about what they're doing that's different from what I'm doing, because that might be able to give me suggestions. If somebody with my problems and those kinds of things, [...] what they're able to do to correct that.

\section{Motivation}

Nearly all participants anticipated enhanced motivation (subtheme) by either upward goal-based comparisons (eg, information that a measure of their diabetes health status was not at goal) or upward social comparisons 


\begin{tabular}{|c|c|}
\hline Characteristic & $\mathrm{n}=25$ \\
\hline Age, mean (range) & $65(45-81)$ \\
\hline \multicolumn{2}{|l|}{ Gender } \\
\hline Female, n (\%) & $13(52)$ \\
\hline Male, n (\%) & $12(48)$ \\
\hline \multicolumn{2}{|l|}{ Race/ethnicity } \\
\hline Black/African-American, n (\%) & $6(24)$ \\
\hline White/Caucasian, n (\%) & $17(68)$ \\
\hline Asian, n (\%) & $2(8)$ \\
\hline \multicolumn{2}{|l|}{ Education } \\
\hline No high school degree, n (\%) & $1(4)$ \\
\hline High school degree/GED, n (\%) & $6(24)$ \\
\hline Some college/associate's degree, n (\%) & $5(20)$ \\
\hline College degree, $\mathrm{n}(\%)$ & $6(24)$ \\
\hline Postgraduate degree, n (\%) & $7(28)$ \\
\hline \multicolumn{2}{|l|}{ Marital status } \\
\hline Single & $7(28)$ \\
\hline Married & $6(24)$ \\
\hline Divorced & $8(32)$ \\
\hline Widowed & $4(16)$ \\
\hline \multicolumn{2}{|l|}{ Scales } \\
\hline $\begin{array}{l}\text { Diabetes numeracy, }{ }^{*} \text { mean \% correct } \\
\text { (range) }\end{array}$ & $53.6 \%(0 \%-100 \%)$ \\
\hline Health literacy, $†$ mean score (range) & $12.4(9-15)$ \\
\hline Diabetes self-efficacy, $\ddagger$ mean (range) & $28.8(21-40)$ \\
\hline HgbA1C, mean & 7.5 \\
\hline \multicolumn{2}{|l|}{ HgbA1C Group } \\
\hline$<7, \mathrm{n}(\%)$, (A1c value range) & 14 (56), (5.6-6.9) \\
\hline 7 to $8.9, \mathrm{n}(\%),(\mathrm{A} 1 \mathrm{c}$ value range) & 7 (28), (7.1-8.4) \\
\hline$>9, \mathrm{n}(\%),(\mathrm{A} 1 \mathrm{c}$ value range) & 4 (16), (10.1-12.6) \\
\hline \multicolumn{2}{|l|}{ Medications } \\
\hline Non-insulin dependent, n (\%) & $12(48)$ \\
\hline Insulin dependent, n (\%) & $13(52)$ \\
\hline \multicolumn{2}{|l|}{ Comorbidities§ } \\
\hline Hyperlipidemia, n (\%) & $17(71)$ \\
\hline Hypertension, n (\%) & $15(60)$ \\
\hline $\begin{array}{l}\text { Atherosclerotic cardiovascular disease, } \\
\mathrm{n}(\%)\end{array}$ & $5(20)$ \\
\hline
\end{tabular}

*Possible score range: 0\% (worst) to 100\% (best).

†Possible score range: 3 (worst) to 15 (best).

†Possible score range: 8 (worst) to 40 (best).

$\S$ Comorbidities are not mutually exclusive, percentages may

total greater than $100 \%$.

GED, general education diploma.

(eg, information that other patients like them were on average doing better).

[If my blood sugar was higher than others like me,] it would definitely motivate me to do better.

Some participants anticipated their motivation would vary depending on the specific measure of health status
Table 2 Themes and subthemes from patient semistructured interviews regarding social and goal-based comparisons of diabetes health status

\begin{tabular}{|c|c|}
\hline Main themes & Subthemes \\
\hline \multirow[t]{4}{*}{ Self-relevance } & Importance \\
\hline & Unique experience of disease \\
\hline & Personalized information* \\
\hline & Similarities to comparison target* \\
\hline \multirow[t]{6}{*}{ Motivation } & Enhanced motivation \\
\hline & Competitiveness* \\
\hline & Resignation or diminished motivation \\
\hline & Perceived self-efficacy \\
\hline & Sustained motivation* \\
\hline & Motivation unchanged \\
\hline \multirow[t]{3}{*}{ Self-concept } & Self-evaluation \\
\hline & Negatively affected \\
\hline & Positively affected \\
\hline \multirow[t]{2}{*}{ Emotions } & Negative emotions \\
\hline & Positive emotions \\
\hline \multirow[t]{5}{*}{ Information seeking } & Self-care information \\
\hline & Medication information \\
\hline & Online as source \\
\hline & Health professional as source \\
\hline & Other patients as source* \\
\hline \multirow[t]{3}{*}{ Medical care } & Appointment and referrals \\
\hline & Medical services \\
\hline & Straightforwardness \\
\hline \multirow[t]{2}{*}{ Self-care } & Enhanced self-care \\
\hline & Diminished self-care \\
\hline
\end{tabular}

*Unique to social comparisons.

(eg, A1C, blood pressure and diabetic eye exam in the last year) involved in the comparison. Distinctly, three participants framed their anticipated motivation from upward social comparison information in terms of competitiveness (subtheme).

[I] t would encourage me to try to do better. Like I said, we all have competitiveness in us and to see that everybody else is winning the race and you're in last place.

Two participants anticipated feeling a sense of resignation (subtheme) at finding out their hemoglobin A1C was not at goal (upward comparison).

I think the only thing I have to fight is resignation to the situation. Why not take another cookie?

Two participants anticipated social and goal-based comparisons would have no impact on their motivation (subtheme) and framed their lack of motivation in terms of their perceived self-efficacy (subtheme).

I'm not able to do anything really active to counteract it.

I take it like it's a fact of life type of thing. 
Six participants anticipated sustained motivation (subtheme) that was unique to downward social comparisons as opposed to goal-based comparisons.

[I]f mine's better, I'd want to keep on making sure it stays better.

\section{Self-concept}

Nearly half of the participants $(n=12)$ anticipated engaging in self-evaluation (subtheme) in response to both upward social and goal-based comparison information related to lifestyle-sensitive measures of diabetes health status (eg, hemoglobin A1C and cholesterol).

I'd be thinking [...] what kind of food changes I need to make. Yeah, if my numbers were not as good or better than anybody [...] that would motivate me to seek out what I needed to do next.

A few participants $(n=5)$ anticipated their self-evaluation in response to upward social and goal-based comparisons would negatively affect their self-concept or beliefs one holds about oneself (subtheme).

I would feel somewhat disappointed in myself that I am not doing the things that I am supposed to be doing.

Two participants anticipated that downward comparisons would positively affect their self-concept (subtheme).

That would make [me] feel good [...] like a winner, accomplished.

\section{Emotions}

Participants anticipated both positive and negative emotional reactions to social and goal-based comparisons of measures of their diabetes health status depending on the direction of the comparison, the type of measure, and their medical history. Emotional responses were anticipated almost exclusively to comparisons regarding the lifestyle-sensitive measures of diabetes health status compared with preventative care measures (eg, diabetic eye exam within the last 12 months).

Eighteen participants anticipated negative emotional responses (subtheme) to upward social and goal-based comparisons related to lifestyle-sensitive measures.

When I have to see that red number it makes me sad. Especially if it's a big change.

Eleven participants also anticipated positive emotional responses (subtheme) to downward social and goal-based comparisons related to lifestyle-sensitive measures.

That'd make you feel good about yourself and happy, like you're a winner.

Some participants explained their anticipated emotional response to upward comparisons of specific lifestyle-sensitive measures of diabetes health status (eg, blood pressure) in terms of the perceived self-relevance of the measure. For example, one participant whose mother had hypertension and had suffered a myocardial infarction described how an upward comparison of her blood pressure would be particularly emotionally resonant.

[S] he was about 72, had the widow-maker heart attack, and her blood pressure was through the roof. [...] Yeah, with what went on [with her], I won't say it would freak me out, but it would grab my attention.

\section{Information seeking}

Slightly more than half of the participants $(n=13)$ anticipated seeking information about self-care (subtheme) and medication options (subtheme) in response to both upward social and goal-based comparisons.

I would be talking to my doctor. I'd be thinking about what kind of drug changes I need to make. What kind of food changes I need to make. Yeah, if my numbers were not as good as anybody in the room that would motivate me to seek out what I needed to do next.

In response to both upward social and goal-based comparisons, some participants also anticipated seeking information from a healthcare professional such as their primary care physician (subtheme) while others anticipated looking online (subtheme).

I would need to look online and see what I can do to reduce [my blood sugar].

If I were higher than average in my group, then I would probably want to talk again to Dr. about what do I need to do.

In response to upward social comparisons, some participants anticipated a desire to learn from individuals similar to them who were doing better (subtheme).

I would want to know how these people are maintaining. If [they are] lower than $\mathrm{X}$, what are the things that they are doing to get to that?

\section{Medical care}

Eighteen participants anticipated seeking medical care in response to both social and goal-based comparisons including scheduling an appointment or referral (subtheme) or medical services such vaccinations (subtheme).

I would probably set an appointment sooner than the regular period.

In contrast to upward comparisons for lifestyle-sensitive measures of diabetes health status, some participants anticipated that upward comparisons for diabetes preventative services would be more straightforward to address (subtheme).

I'd go get the [vaccination]. That's the simplest thing.

\section{Self-care}

Finally, 10 participants anticipated enhanced self-care (subtheme) in response to upward social and goal-based 
comparisons including improving their diet, exercise, and adherence to medications.

I would definitely modify my diet and probably pick up my exercise a little bit.

One participant anticipated diminished self-care (subtheme) in response to upward comparisons of lifestyle sensitive measures of diabetes health status.

I'd probably say, 'The hell with it. I might as well eat what I want.' That's what I would think.

\section{DISCUSSION}

Our findings provide significant insights into the anticipated cognitive and behavioral responses of patients with T2DM to receiving social and goal-based comparison information regarding their diabetes health status. Prior studies have focused on understanding the social comparisons patients make on their own and the impact of forced comparisons with a specific target, typically a hypothetical patient, on affect, motivation, and self-care. ${ }^{1315} 16$ 32-34 Our study is unique in examining patients' desire and anticipated response to receiving social comparison information in a clinical context involving actual patients and how receiving such information would compare to typical goal-based comparisons.

Many patients desired social comparison information and may perceive it as more personalized than goal-based comparisons. Our findings suggest that the perception of similarity (ie, how much the individual identifies with the target) may drive both the perceived self-relevance of the information and its perception as more personalized. Researchers have noted that perceived similarity with the comparison target may decrease psychological distance between the self and the target thereby increasing self-relevance and the ability of the comparison to impact behavioral intention. ${ }^{1435}$ In particular, participants noted the importance of being compared with others within their same age group, gender, racial and ethnic group, on similar medications, and with similar body types. In their study of patients with T2DM who read one of four brief descriptions of a patient with T2DM, Arigo and colleagues $^{34}$ also noted that the perception of similarity strongly mediated the impact of the comparison on motivation for diabetes self-care behaviors.

Patients in our study anticipated increased motivation from both upward social and goal-based comparisons, and noted that social comparisons, in particular, may be motivating by engaging patients' competitiveness. While other researchers have found that downward comparisons can result in decreased motivation, ${ }^{34}$ the patients in our study often anticipated downward comparison information would sustain their motivation for self-care. Patients who anticipated not being motivated by upward comparisons either described social comparison information as irrelevant to their unique experience of diabetes or described feeling resigned to their current diabetes health status and a lack of confidence in their ability to effect change (ie, low self-efficacy). Our findings highlight the potential modifying effects that the direction of the comparison, perceived self-relevance, and perceived self-efficacy (see conceptual model, figure 1) may have on patients' motivation in response to comparison information. Our findings extend those of Schokker and colleagues $^{15}$ who found that patients highly focused on obtaining positive outcomes (high promotion-focused), as opposed to those focused on preventing a bad outcome (high prevention-focused), reported more motivation when confronted with an upward comparison with another patient with diabetes.

In our study, participants anticipated that both social and goal-based comparison information would promote self-evaluation. This is consistent with Thorton and Arrowood's ${ }^{36}$ description of self-evaluation as one of the main processes that underlies social comparison. Participants also anticipated that engaging in self-evaluation would affect self-concept, with upward comparisons potentially negatively affecting self-concept and downward comparisons positively affecting self-concept. In addition, participants anticipated upward comparisons would lead to negative emotions and downward comparisons would lead to positive emotions. This is consistent with other work describing downward comparisons as affording patients a sense of well-being and empowerment. ${ }^{32}$ Participants who anticipated upward comparisons would lead to negative emotions frequently also described an anticipated increase in their motivation to change the way they manage their diabetes. Negative affect may inform individuals that something is wrong and that they are not achieving their aims and that they should not be satisfied with their performance. ${ }^{37}$ Many studies support cognitive responses to negative emotions resulting in improved behavior or performance. ${ }^{38} 39$

Participants in our study anticipated behaviors aimed at improving their diabetes health status in response to upward comparisons. Notably, when considering how they would respond to upward social comparisons, participants anticipated a desire for information from above average members of their cohort that they could use to improve their health status. This highlights a potentially unique aspect of providing such information to patients. Social comparisons have been shown to be particularly salient in a variety of chronic disease for promoting and maintaining health behaviors. ${ }^{32} 40$

Our findings suggest that providing patients with diabetes with social and goal-based comparison information regarding a variety of measures of their diabetes health status may affect motivation, mood, and self-concept in ways that may improve or sustain diabetes self-care behaviors for some patients. Social comparison information in particular may be viewed as more personalized, uniquely engage some patients' competitiveness, and drive more information seeking. Several interventions have sought to increase patients' motivation and confidence to manage their diabetes ${ }^{41}{ }^{42}$; however, progress 
has been limited, and the need for effective, scalable and sustainable interventions persists. ${ }^{41}{ }^{43}$ Strategies such as the 'know your numbers' campaign (promoting patient knowledge of their actual and target health outcomes such as HgbA1C values) have had mixed success in increasing the confidence and motivation necessary to improve patients' diabetes self-management. ${ }^{10-12}{ }^{44}$ To be effective, strategies to provide information to patients must be combined with other behavioral strategies to motivate and assist patients in managing their diabetes. ${ }^{10} 12434546$ The recent push toward electronic health record adoption and the development of patient web portals provide a new opportunity for healthcare systems and patients to view, share, and take action in response to health data and information. How to optimally use these new technologies to improve patient care remains an active area of research. ${ }^{47}$ Despite evidence that social comparison information may increase motivation and is central to the patient experience, ${ }^{15} 28-3032$ it has been underused to modify health behaviors in patients with diabetes or other chronic medical conditions. Healthcare systems may consider leveraging patient-facing technologies such as patient web portals to provide patients with social and goal-based comparisons of their diabetes health status alongside other features aimed at increasing patient engagement and facilitating information seeking and self-care (eg, educational modules, secure messaging and text reminders). ${ }^{47-50}$ Further research is needed to determine how to best share social and goal-based comparisons with patients, which patients are most likely to benefit, and whether patients' anticipated responses match actual responses to this information in practice as well as the impact of sharing this information on cognitive, behavioral, and clinical outcomes.

Our study has some limitations. Participants were limited to a convenience sample drawn from a single academic primary care clinic, thus our findings may not be generalizable to other settings. Although our sample was heterogeneous across a number of different sociodemographic and clinical variables, it was not fully representative the overall population of patients with T2DM in the USA. In addition, our sample size was not large enough to assess differences based on sociodemographic factors such as race/ethnicity, education, and income. Participants electing to participate in research may be inherently more motivated, which could result in selection bias. Participants answered questions in the setting of one-on-one in-person interviews, and social desirability bias may have impacted our results. Moreover, participants were asked how they would respond to hypothetical scenarios, and their actual response may vary from their anticipated response.

Despite these limitations, our study highlights a potentially significant strategy for increasing patient motivation and improving diabetes care. Providing patients with social and goal-based comparison information regarding their diabetes health status has the potential to be an effective and scalable approach to promoting diabetes self-care behaviors. Further research is needed to determine how to deliver this information reliably to patients and its impact on diabetes care.

Acknowledgements We wish to thank Tiffany L Israel, MSSW, Community Navigator, at Vanderbilt Institute for Medicine and Public Health for her help interviewing participants for this study.

Contributors WM, KAW, and TAE conceived of the study. WM, KAW, DGS, GBH, and TAE designed the study. WM and RJT recruited patients. WM and RJT collected the data. WM, DGS, KRB, and RJT analyzed the data. All authors interpreted the data. WM drafted the manuscript. All authors contributed substantially to its revision. WM takes responsibility for the paper as a whole.

Funding This work was supported by NIDDK/NIH K23DK106511 and 2P30DK092986-07 and NCATS/NIH UL1 TR000445.

\section{Competing interests None declared.}

Patient consent Not required.

Ethics approval The Vanderbilt University Institutional Review Board approved this research.

Provenance and peer review Not commissioned; externally peer reviewed. Data sharing statement № additional data are available.

Open Access This is an Open Access article distributed in accordance with the Creative Commons Attribution Non Commercial (CC BY-NC 4.0) license, which permits others to distribute, remix, adapt, build upon this work non-commercially, and license their derivative works on different terms, provided the original work is properly cited and the use is non-commercial. See: http://creativecommons.org/ licenses/by-nc/4.0/

(c) Article author(s) (or their employer(s) unless otherwise stated in the text of the article) 2018. All rights reserved. No commercial use is permitted unless otherwise expressly granted.

\section{REFERENCES}

1. Centers for Disease Control Prevention. National diabetes statistics report: estimates of diabetes and its burden in the United States 2017. Atlanta, GA: US Department of Health and Human Services, 2017.

2. Powers MA, Bardsley J, Cypress M, et al. Diabetes self-management education and support in type 2 diabetes: a joint position statement of the American Diabetes Association, the American Association of Diabetes Educators, and the Academy of Nutrition and Dietetics. The Diabetes Educator 2017:43:40-53.

3. Shrivastava SR, Shrivastava PS, Ramasamy J. Role of self-care in management of diabetes mellitus. J Diabetes Metab Disord 2013;12:14

4. Yu CH, Parsons JA, Mamdani M, et al. A web-based intervention to support self-management of patients with type 2 diabetes mellitus: effect on self-efficacy, self-care and diabetes distress. BMC Med Inform Decis Mak 2014;14:117.

5. Bolen SD, Chandar A, Falck-Ytter C, et al. Effectiveness and safety of patient activation interventions for adults with type 2 diabetes: systematic review, meta-analysis, and meta-regression. J Gen Intern Med 2014;29:1166-76.

6. Vorderstrasse A, Lewinski A, Melkus GD, et al. Social support for diabetes self-management via ehealth interventions. Curr Diab Rep 2016;16:56.

7. Fisher EB, Boothroyd RI, Elstad EA, et al. Peer support of complex health behaviors in prevention and disease management with specia reference to diabetes: systematic reviews. Clin Diabetes Endocrinol 2017;3:4.

8. Qi L, Liu Q, Qi X, et al. Effectiveness of peer support for improving glycaemic control in patients with type 2 diabetes: a meta-analysis of randomized controlled trials. BMC Public Health 2015;15:471.

9. Wiebe DJ, Helgeson V, Berg CA. The social context of managing diabetes across the life span. Am Psychol 2016;71:526-38.

10. Stark Casagrande S, Ríos Burrows N, Geiss LS, et al. Diabetes knowledge and its relationship with achieving treatment recommendations in a national sample of people with type 2 diabetes. Diabetes Care 2012;35:1556-65.

11. Berikai P, Meyer PM, Kazlauskaite R, et al. Gain in patients' knowledge of diabetes management targets is associated with better glycemic control. Diabetes Care 2007;30:1587-9. 
12. Heisler M, Piette JD, Spencer M, et al. The relationship between knowledge of recent $\mathrm{HbA1} \mathrm{c}$ values and diabetes care understanding and self-management. Diabetes Care 2005;28:816-22.

13. Brakel TM, Dijkstra A, Buunk AP, et al. Impact of social comparison on cancer survivors' quality of life: an experimental field study. Health Psychol 2012;31:660-70.

14. Buunk BP, Gibbons FX, Buunk A. Health, coping, and well-being: perspectives from social comparison theory: Psychology Press, 2013.

15. Schokker MC, Keers JC, Bouma J, et al. The impact of social comparison information on motivation in patients with diabetes as a function of regulatory focus and self-efficacy. Health Psychol 2010;29:438-45

16. Arigo D, Suls JM, Smyth JM. Social comparisons and chronic illness: research synthesis and clinical implications. Health Psychol Rev 2014;8:154-214.

17. Schunk DH. Developing children's self-efficacy and skills: The roles of social comparative information and goal setting. Contemp Educ Psychol 1983;8:76-86.

18. Murphy KR, Cleveland J. Understanding performance appraisal: social, organizational, and goal-based perspectives: Sage, 1995.

19. Ritchie J, Lewis J, Nicholls CM, et al; Qualitative research practice: a quide for social science students and researchers: Sage, 2013.

20. United States Census Bureau. QuickFacts. Davidson County, Tennessee, 2016. https://www.census.gov/quickfacts/fact/table/davi dsoncountytennessee/PST045216. (accessed 1 Feb 2018).

21. Sarkar U, Schillinger D, López A, et al. Validation of self-reported health literacy questions among diverse English and Spanishspeaking populations. J Gen Intern Med 2011;26:265-71.

22. Huizinga MM, Elasy TA, Wallston KA, et al. Development and validation of the Diabetes Numeracy Test (DNT). BMC Health Serv Res 2008;8:96.

23. Wallston KA, Rothman RL, Cherrington A. Psychometric properties of the perceived diabetes self-management scale (PDSMS). J Behav Med 2007;30:395-401.

24. Guest G, Bunce A, Johnson L. How many interviews are enough? An experiment with data saturation and variability. Field methods 2006;18:59-82.

25. Francis JJ, Johnston $\mathrm{M}$, Robertson $\mathrm{C}$, et al. What is an adequate sample size? Operationalising data saturation for theory-based interview studies. Psychol Health 2010;25:1229-45.

26. Miles MB, Huberman AM, Saldana J. Qualitative data analysis. Sage 2013

27. Glaser B, Strauss AL. The discovery of grounded theory: strategies for qualitative research. Chicago: Aldine, 1967.

28. Suls JE, Wheeler LE. Handbook of social comparison: theory and research: Kluwer Academic Publishers, 2000.

29. Taylor SE, Wayment HA, Carrillo M. Social comparison, selfregulation, and motivation, 1996.

30. Veroff J. Social comparison and the development of achievement motivation. achievement-related motives in children, 1969:46-101.

31. Scholer AA, Ozaki Y, Higgins ET. Inflating and deflating the self: sustaining motivational concerns through self-evaluation. J Exp Soc Psychol 2014;51:60-73.

32. Gorawara-Bhat $\mathrm{R}$, Huang ES, Chin $\mathrm{MH}$. Communicating with older diabetes patients: self-management and social comparison. Patient Educ Couns 2008;72:411-7.
33. Arigo D, Smyth JM. Guided social comparisons differentiate motivation to improve health behaviors. Annals of Behavioral Medicine 2014;47:s134.

34. Arigo D, Smyth JM, Suls JM. Perceptions of similarity and response to selected comparison targets in type 2 diabetes. Psychol Health 2015;30:1206-20.

35. Mussweiler T. Comparison processes in social judgment: mechanisms and consequences. Psychol Rev 2003;110:472-89.

36. Thornton DA, Arrowood AJ. Self-evaluation, self-enhancement, and the locus of social comparison. J Exp Soc Psychol 1966;1:40-8.

37. De Cremer D, Zeelenberg M, Murnighan JK. Social psychology and economics: Psychology Press, 2013.

38. Forgas JP, worry Don't. Don't Worry, Be Sad! On the cognitive, motivational, and interpersonal benefits of negative mood. Curr Dir Psychol Sci 2013;22:225-32.

39. Juhl J, Routledge C, Hicks JA, et al. Can affectively negative experiences contribute to well-being? The Affectively Negative Need-Fulfillment Model: The Happy Mind: Cognitive Contributions to Well-Being, Springer 2017:389-407.

40. Suls J. Contributions of social comparison to physical illness and well-being. Social psychological foundations of health and illness 2003:226-55.

41. Pal K, Eastwood SV, Michie S, et al. Computer-based interventions to improve self-management in adults with type 2 diabetes: a systematic review and meta-analysis. Diabetes Care 2014;37:1759-66.

42. Sacks RM, Greene J, Hibbard J, et al. Does patient activation predict the course of type 2 diabetes? A longitudinal study. Patient Educ Couns 2017:100:1268-75.

43. Hibbard JH, Greene J. What the evidence shows about patient activation: better health outcomes and care experiences; fewer data on costs. Health Aff 2013;32:207-14.

44. Iqbal $\mathrm{N}$, Morgan $\mathrm{C}$, Maksoud $\mathrm{H}$, et al. Improving patients' knowledge on the relationship between $\mathrm{HbA} 1 \mathrm{c}$ and mean plasma glucose improves glycaemic control among persons with poorly controlled diabetes. Ann Clin Biochem 2008;45:504-7.

45. Serrano-Gil M, Jacob S. Engaging and empowering patients to manage their type 2 diabetes, Part I: a knowledge, attitude, and practice gap? Adv Ther 2010;27:321-33.

46. Willaing I, Rogvi SA, Bøgelund M, et al. Recall of HbA1c and self-management behaviours, patient activation, perception of care and diabetes distress in Type 2 diabetes. Diabet Med 2013;30:e139-e142.

47. Osborn CY, Mayberry LS, Mulvaney SA, et al. Patient web portals to improve diabetes outcomes: a systematic review. Curr Diab Rep 2010;10:422-35.

48. Mishuris RG, Yoder J, Wilson D, et al. Integrating data from an online diabetes prevention program into an electronic health record and clinical workflow, a design phase usability study. BMC Med Inform Decis Mak 2016;16:88.

49. Nelson LA, Mulvaney SA, Gebretsadik T, et al. The MEssaging for Diabetes (MED) intervention improves short-term medication adherence among low-income adults with type 2 diabetes. J Behav Med 2016;39:995-1000.

50. Kuo A, Dang S. Secure Messaging in Electronic Health Records and Its Impact on Diabetes Clinical Outcomes: A Systematic Review. Telemed J E Health 2016;22:769-77. 